\title{
VAlidation of the Orientation to Life Questionnaire (OLQ) IN A CHEMICAL FACTORY
}

\author{
LOURENS VAN SCHALKWYK \\ SEBASTIAAN ROTHMANN \\ WorkWell: Research Unit for People, Policy and Performance \\ North-West University \\ South Africa
}

Correspondence to: Sebastiaan Rothmann

e-mail: ian@ianrothmann.com

\begin{abstract}
The objectives of this study were to assess the psychometric properties of the Orientation to Life Questionnaire (OLQ) and to investigate the differences in sense of coherence among demographic groups in a chemical factory environment. The OLQ and a biographical questionnaire were administered to chemical factory employees $(N=583)$. A three-factor model (manageability, meaningfulness and comprehensibility) was confirmed through structural-equation modelling. From the original 29-item OLQ, an 11-item OLQ and a 6-item OLQ were first derived with good fit statistics. With the 11-item OLQ, significant differences in sense of coherence among some demographic groups viz. qualification and job level were then confirmed.
\end{abstract}

Keywords: sense of coherence, Orientation to Life Questionnaire (OLQ), validity, reliability, employee interaction

Increasing complexity in the workplace and the resulting demands on employees have led to a significant volume of research on work-related distress and coping behaviour (Geyer, 1997). More recently, research focus has shifted to personal control and the relationship among social factors, health and illness (Lundberg \& Nyström Peck, 1994) or the strengths that people can apply to protect themselves from falling ill under stressful conditions (Geyer, 1997). Various approaches applied in an attempt to address this research issue exist, notably the "hardy personality" of Kobasa (1979), the "self-efficacy" of Bandura (1982) and the "sense of coherence" of Antonovsky (1987, 1993). Antonovsky's salutogenic model, however, has drawn much attention in contemporary studies regarding the prevention and promotion of health (Lindfors, Lundberg \& Lundberg, 2005; Lundberg \& Nyström Peck, 1994; Söderfeldt, Söderfeldt, Ohlson, Theorell \& Jones, 2000). Sense of coherence appears to covary strongly with health (Geyer, 1997; Larsson \& Kallenberg, 1999).

The working environment is changing at an unprecedented speed. The line between work and home as well as where and when work occurs has become blurred. People are often expected to work long hours or to be available $24 / 7$, and the number of dual-income families has increased (Colteryahn \& Davis, 2004). The result is that people experience an imbalance between workplace demands and the resources that they have in order to cope with these demands. Amid similar stressful situations, some individuals, however, find it difficult to keep up with the pace while others tend to cope better (Ortlepp \& Friedman, 2001).

The difference between people who cope and those who do not cope has been well researched in an attempt to identify the driving forces and strengths that enable a person to meet and handle life's adversities while maintaining or regaining health (Nygren et al., 2005). A common theme reported by Lundberg and Nyström Peck (1994) is that an individual's cognition, combined with his/her view on the environment, including the accompanying reaction to it, is important in coping with stress. Lindsfors et al. (2005) explain differences in the coping and non-coping of individuals in terms of sense of coherence. Individuals who do not cope well tend to view the world as unorganised and meaningless and tend not to have resources to deal with daily life, while those who cope view the world as organised and meaningful and have sufficient resources to meet life's demands.

Sense of coherence develops during early adulthood (Feldt, Leskinen, Kinnunen \& Ruoppila, 2003), which coincides with the first employment years, when greater psychological stability, independence and a sense of identity are established (Antonovsky, 1987; Antonovsky \& Sagy, 2001). During this phase, young adults commit themselves to life, marriage, a career, a particular lifestyle and social roles and they form a world-view (Feldt, Kivimäki, Rantala \& Tolvanen, 2004). From early middle age (30 years and older) - what Larsson and Kallenberg (1999) call the "formative years" - this sense of coherence is accepted to be relatively stable onwards in life (Eriksson \& Lindström, 2005; Nilsson, Holmgren, Stegmayer \& Westman, 2003).

Sense of coherence (Antonovsky, 1979) is defined as a global orientation expressing a person's pervasive and enduring feeling of confidence that (1) the stimuli deriving from one's internal and external environments in the course of living are structured, predictable and explicable [comprehensibility], that (2) the resources available to one meet the demands posed by these stimuli [manageability] and that (3) these demands are challenges worthy of investment and engagement [meaningfulness]. An individual's sense of coherence is proposed to develop in relation to his/her experience of the world as predictable and consistent as well as the ability to shape life's outcomes (Ortlepp \& Friedman, 2001). Sense of coherence is a generalised orientation towards the environment, which serves as a source of general resistance resources for an individual to draw on in order to deal with the demands of life (Nilsson et al., 2003).

Sense of coherence is not a personality trait but an enduring person and view-of-the-world-related characteristic that influences appraisals of meaning ascribed to different situations (Flannery, Perry, Penk \& Flannery, 1994; Larsson \& Kallenberg, 1996, 1999). Antonovsky $(1987,1993)$ describes sense of coherence as a dispositional personality orientation, which Hanse and Engström (1999), in turn, highlight as important in perceiving and controlling the environment for meaningful and appropriate action. 
The importance of sense of coherence for an individual to cope with daily life demands is well documented. Lindfors et al. (2005) point out that the complexities of daily living and being confronted with various contradictory stimuli form a fundamental assumption in the conceptualisation of sense of coherence. They stress the importance of the three sense-ofcoherence components - comprehensibility, manageability and meaningfulness - for an individual to deal successfully with everyday stimuli and in the promotion of health (Geyer, 1997). In addition, the relationship between sense of coherence and illness or health is also well documented (Antonovsky, 1993, Larsson \& Kallenberg, 1999; Lundberg \& Nyström Peck, 1994).

Research on sense of coherence has been criticised for three reasons, namely the theoretical sense-of-coherence construct, the instruments of and adherence to psychometric principles, and inadequate statistical techniques used in analysing data. These criticisms stem primarily from an apparent lack of conceptual clarity about the sense-of-coherence construct and a lack of empirical evidence to support the theoretical threefactor structure (Larsson \& Kallenberg, 1999; Schumann, Hapke, Meyer, Rumpf \& John, 2003). This is evident from the psychometric properties of the OLQ (Lundberg \& Nyström Peck, 1994; Schumann et al., 2003), where Antonovsky's theoretical three-factor sense-of-coherence construct is ignored, resulting in some of the underlying constructs (factors) being discarded in favour of better fit results. This deviation from Antonovsky's theoretical sense-of-coherence construct is taking place without an alternative theory or model being presented for sense of coherence. Finally, statistical inadequacies are reported with the interpretation of OLQ data as a multifaceted personality construct (Hittner, 2000). Despite these concerns, however, sense of coherence continues to interest researchers (Feldt et al. 2004) and various attempts have been made to test the stability of sense-of-coherence measures (Smith, Breslin \& Beaton, 2003). These include attempts to confirm the sense-of-coherence structure through Structural Equation Modelling (SEM) (Larsson \& Kallenberg, 1999), the revisiting of some conceptual considerations (Geyer, 1997) and the development of various forms of an OLQ questionnaire for different applications, such as paper-and-pen administration or interview settings (Lundberg \& Nyström Peck, 1994; Randall, 2006; Schumann et al., 2003).

Sense of coherence is conceptualised based on three subconstructs, namely comprehensibility, manageability and meaningfulness (Antonovsky, 1987, 1993). Although Antonovsky (1987) regards comprehensibility as a cognitive dimension, manageability as an instrumental dimension and meaningfulness as a motivational dimension, he also stresses the high interdependence of the three sense-of-coherence components and cautions against the separate use of the three sub-scales.

While widely used and recommended for inclusion in surveys, sense of coherence as a concept and the accompanying OLQ measurement tools are frequently criticised for various reasons. The usefulness of the sense-of-coherence construct, however, is widely supported, yet researchers are frequently warned against various aspects of sense of coherence and the OLQ. In the more than three decades since Antonovsky presented the sense-of-coherence concept and the OLQ, various attempts have been made to address at least some of the concerns. These attempts include specific psychometric alterations, such as item selection and statistical attempts, to increase the validity of the original OLQ and shorter versions thereof. These attempts, although commendable, do not seem to have resulted in better prospects for the sense-of-coherence construct or the OLQ questionnaire.

Finding an answer to the problems associated with sense of coherence and the measurement of sense of coherence will require focus on improving the psychometric properties of OLQ formats and on statistical techniques applied to the analysis of sense-of-coherence scales - all within the ambit of Antonovsky's initial conceptual framework. The purpose of this study was to test the psychometric properties of the original OLQ-29 format in a multilingual setting and to construct a shortened version of the OLQ, with more than one question for each of the three initial sense-of-coherence constructs.

\section{Conceptual considerations}

Despite its wide acceptance and application, sense of coherence has been criticised for a lack of conceptual clarity and a lack of empirical evidence to support the three-factor structure (Larsson \& Kallenberg, 1999; Schumann et al., 2003). While, on a theoretical level, Antonovsky's three-factor construct for sense of coherence is accepted, Antonovsky $(1987,1993)$ himself cautions against using the three factors as sub-scales. Focus is therefore now tending towards a one-factor approach in constructing sense-of-coherence measurement scales (OLQ), such as in Schumann et al. (2003).

To date, several studies analysing sense-of-coherence scale factors have failed to identify the three components of sense of coherence. According to Schumann et al. (2003), Principle Component Analysis has produced only a single-factor solution (Antonovsky, 1993) and Confirmatory Factor Analysis has produced many more factors that can only be distantly equated to components posited by Antonovsky. Some researchers, viz. Lundberg and Nyström Peck (1994), however, maintain the three-factor construct by selecting one item per factor when they construct their three-item OLQ. Hittner (2000) points at the highly intercorrelated nature of the three sense-of-coherence factors as a multifaceted personality construct with multiple subordinate constructs that are usually intercorrelated factors or sub-scales (superordinate constructs).

Although research further tends to focus primarily on a total sense of coherence (one factor) (Hittner, 2000), research regarding suicidal ideation during different periods of hospitalisation using the factors as predictors hints at the usefulness of the separate sub-scales in practice (Petrie \& Brooks, 1992). Both theory (Antonovsky, 1987) and research (Petrie \& Brooks, 1992) suggest that the three factors might yield differential predictive associations. In the absence of an alternative theoretical model, the notion of sense of coherence as a unidimensional-factor construct should be considered, although a three-factor senseof-coherence construct is theoretically posited and supported by practical research

Another difficulty with Antonovsky's sense of coherence is the posited relative stability of sense of coherence after three decades. Snekkevik, Anke, Stanghelle and Fugl-Meyer (2003) report that, although median OLQ scores (sense-of-coherence levels) were found to be relatively stable over time, individual scores sometimes showed large variations. Furthermore, while Nygren et al. (2005) found sense of coherence to be equivalent or higher for older groups compared to their younger counterparts, Feldt et al. (2003) could not find the same stability in sense of coherence in older subjects compared to younger ones. The development of sense of coherence is a result of new life experiences (Sjöström, Langius-Eklof \& Hjertberg, 2004). Life experiences, however, do not end at the age of 30 and, although the relative stability of sense of coherence after 30 years of age is accepted, Feldt et al. (2004) posit that sense of coherence can be expected to change if it coincides with major life changes. These significant life changes are equated to the initiation of new life experiences for the individual, such as giving birth for the first time (Nilsson et al., 2003). Women who were about to give birth a second time reportedly had higher levels of sense of coherence than did women expecting a first child, supporting the notion that new life experiences contribute to sense of coherence (Sjöström et al., 2004). Although an occasional life event might alter the sense-of-coherence strength, it might be temporary 
and the subject is likely eventually to return to his/her original sense-of-coherence level. This supports findings by Antonovsky (1987) that sense of coherence is relatively stable in adulthood except when radical events occur in a person's life. Feldt et al. (2003) do not find that age plays a significant role in the stability, level or mean changes in sense of coherence and suggest that Antonovsky's theory be revised as far as the stability of sense of coherence in adulthood is concerned.

It can therefore be assumed that, although one major life event can change the strength of sense of coherence, the sense-ofcoherence level can return to a previous level in the absence of more major events in close succession. Depending on the nature, intensity and frequency of these life events, which cannot be unlearned or un-experienced, this collective experience should contribute to an individual's overall sense-of-coherence strength

According to Lundberg and Nyström Peck (1994), sense of coherence is important in any investigation into the social determinants of health and ill health. Sense of coherence has been reported as an important contributing component of one's general health and well-being (Lundberg \& Nyström Peck, 1994). Sense of coherence has been found to mediate the effect of stress on personal efficacy and, while job stress impacts on exhaustion, a strong sense of coherence moderates the effect of job stress on exhaustion (Rothmann, Jackson \& Kruger, 2003), enabling individuals to make the necessary adjustments in life in order to be stable and productive individuals (Strauser \& Lustig, 2003). Petrie and Brook (1992) report on the usefulness of sense of coherence and, more specifically, the usefulness of sense-of-coherence sub-scales (or factors) in predicting initial suicide ideation and reattempting suicide ideation. Anderson and Riger (1991) make reference to the strong association between the sense-of-coherence manageability factor and depression, highlighting the relationship between expectations of personal control and depression.

Sense of coherence has been used successfully in various intervention settings, such as counselling (Olsson \& Hwang, 2002; Strauser \& Lustig, 2003) and group dynamics (Cilliers, 2001). Moreover, it has been linked to psychological and psychosocial constructs, such as well-being and satisfaction with life (Schumann et al., 2003; Volanen, Lahelma, Silventoinen \& Suominen, 2004), burnout and work engagement (Rothmann, Steyn \& Mostert, 2005), coping with stressors and the maintenance of health (Agardh et al., 2003), of stress, of physical and psychological health and of well-being (Holmberg, Thelin \& Stiernström, 2004), and ill health and disease (Antonovsky, 1993; Hanse \& Engström, 1999; Javtokas, Lindström, \& Zagminas, 2004; Larsson \& Kallenberg, 1996; Lundberg \& Nyström Peck 1994; Söderfeldt et al., 2000).

Contradictory findings are also reported regarding the relationship between sense of coherence and demographic variables. Earlier, correlations were reported between sense of coherenceand demographicvariables, suchasgender, age, tenure, education, work function and employment status (Antonovsky \& Sagy, 2001; Hanse \& Engström, 1999; Larsson \& Kallenberg, 1996; Tuomi, Seitsamo \& Huuhtanen, 1999). Holmberg et al. (2004), however, indicate sense of coherence as independent from socio-demographic factors, with low correlations between sense of coherence and socio-demographic variables. Despite the apparent usefulness and general acceptance of the senseof-coherence concept and its related instrument (the OLQ) and the reported relationship to other psychosocial measures, sense of coherence has drawn debate since its inception (Holmberg et al., 2004).

\section{Psychometric and statistical considerations}

The lack of conceptual clarity mentioned by Larsson and Kallenberg (1999) is confirmed by the failure of multivariate statistical techniques, such as factor analysis, to identify the supposed three factors (or components) of sense of coherence. Despite correlation studies validating Antonovsky's sense-ofcoherence scale (Flannery et al., 1994), different multivariate analyses lead to significantly different results. Principle Component Analysis resulted in single-factor solutions, while Confirmatory Factor Analysis resulted in many more factor structures that only distantly equated Antonovsky's conceptualised components (Schumann et al., 2003). In practice, the problematic psychometrics of the OLQ result in various attempts to test and manipulate data by means of SEM with AMOS (Arbuckle, 1997) to find a more suitable model fit that complements the sense-of-coherence concept. With the lack of conceptual clarity, some researchers also opt to ignore Antonovsky's three-factor construct in favour of better fit statistics with a single-factor approach (Schumann et al., 2003).

Another relic of the lack of conceptual clarity and the resulting freedom in research is that item numbers are sacrificed to such an extent that sense-of-coherence instruments are reduced to as few as three items (Lundberg \& Nyström Peck, 1994; Schumann et al., 2003). Lundberg and Nyström Peck (1994) retain Antonovsky's three-factor construct for sense of coherence for the three-item OLQ that they developed for interviewing purposes. The three questions each represent a dimension. They note the impossibility of single questions to grasp every detail of the theoretical intentions behind each of the dimensions (Lundberg \& Nyström Peck, 1994), although they could still capture the essence of sense of coherence in a large survey, while maintaining the three-factor structure with one item per factor. In answer to Lundberg and Nyström Peck (1994), Schumann et al. (2003) use three original OLQ-29 items, viz. items 12 and 19 loading on the comprehensibility factor and item 14 loading on the meaningfulness factor, while discarding the manageability factor in favour of better fit statistics and ultimately reporting only what Hittner (2000) refers to as the total sense of coherence (one-factor structure)

Research indicates a preference for original items from the 29-item OLQ when alternative versions of the OLQ are constructed. The OLQ-13 elects 13 items from the OLQ-29 and Schumann et al. (2003) use three original OLQ-29 items for their version of a three-item OLQ. New items created by Lundberg and Nyström Peck (1994) for interview application and the additional items that Randall (2006) developed in her study need to be tested in multiple-research scenarios to confirm validity and reliability in different settings. On face value, some of the new items do not comply with basic psychometric requirements, such as multiple-phrased items, and complex language syntax, such as parenthesis, pleonasms and double negatives.

The theoretical three-factor structure for sense of coherence is widely accepted but often negated in favour of a one-factor approach in an attempt to better the statistical properties of the sense-of-coherence measurement scales. In the absence of an alternative theoretical model to replace Antonovsky's theory and with research failing to confirm the three-factor structure of sense of coherence, further research should treat the OLQ as a single-factor instrument, while attempts should be made to ensure that all three theoretical factors are covered with items.

The need to develop a shorter version of the OLQ is apparent for three reasons. The original 29-item version (Antonovsky, 1987) was designed to be a paper-and-pencil test. Lundberg and Nyström Peck (1994), however, find the questions to be unsuitable for interview use. The 29-item version is also found to be too long for inclusion in multi-purpose surveys already congested with many other questions, resulting in a choice between excluding a sense-of-coherence measurement and capturing the essence of sense of coherence with as few items as possible (Lundberg \& Nyström, 1994). Lastly, the debate 
about the conceptual structure of sense of coherence prevails without more clarity about a multi or monostructure and the underlying theoretical three-component structure is therefore often negated, resulting in fewer items in favour of better statistical coefficients.

\section{Measurement of sense of coherence}

The most commonly found way to measure sense of coherence is with the 29-item OLQ-29 (Antonovsky, 1987). Several versions of the OLQ are available, varying from the original 29-item scale of Antonovsky (1987) to scales with as little as three items (Lundberg \& Nyström Peck, 1994, Schumann et al., 2003). The general interest in the sense-of-coherence construct is also evident from the fact that the OLQ is available in at least 33 languages in more than 30 countries and in at least 15 versions (Eriksson \& Lindström, 2005; Lundberg \& Nyström Peck, 1994). Five variations of the OLQ were considered for this study, the first being the 29-item version originally designed by Antonovsky (1987), the second a shortened 13-item version derived from the original 29-item version, a 23-item version (Randall, 2006) and two three-item versions, one by Lundberg and Nyström Peck (1994) and one by Schumann et al. (2003). The reason for shorter versions is generally given as these versions being more suitable for large population surveys or where questionnaire batteries are already compounded (Lundberg \& Nyström Peck, 1994; Schumann et al., 2003).

The importance of the three different factors contributing to the sense-of-coherence structure described by Antonovsky (1987) is essential in developing sense of coherence. The same three factors are also repeatedly highlighted in literature as important for an individual to be able to deal with daily demands (Lindfors et al., 2005). The use of sense-of-coherence measures across different languages and cultures has also been reported to produce similar results regarding validity and reliability (Antonovsky, 1993, Lundberg \& Nyström Peck, 1994). Repeated factor analyses of the OLQ scale, however, have failed to identify distinctive separate factors representing the three sense-of-coherence components (Schumann et al., 2003). Different analytical attempts, such as Principle Factor Analysis, repeatedly confirm a single-factor structure as indicated earlier by Antonovsky (1993) (Schumann et al., 2003) or a multi-substructure of sense of coherence with various new factors only remotely comparable to Antonovsky's original components. Two distinct opinions state that sense of coherence is only a one-dimensional construct (Schumann et al., 2003), as the three-component structure cannot be statistically confirmed and the OLQ instruments are restricted in their design and so limited in extracting the theoretically supposed three-component structure of sense of coherence because other statistical methods are inadequately applied to confirm the three-factor structure for sense of coherence.

The first purpose of this study was to analyse the original OLQ-29 items and to test the items using AMOS (Arbuckle, 1997) in different models to come up with a shortened version of the OLQ instrument that complies with the three-factor sense-of-coherence structure as conceptualised by Antonovsky. The second purpose was to explore the differences in senseof-coherence means for different demographic subgroups in a sample taken from the chemical factory environment.

\section{RESEARCH DESIGN}

\section{Research approach}

A cross-sectional survey design was used in this study (Shaughnessy \& Zechmeister, 1997). Questionnaires were used to gather data in a field survey.

\section{Research method}

Participants

The sample drawn was from the employee population from a number of businesses in the chemical factory environment in South Africa. All employees in this specific population were targeted and as many employees as possible were accommodated during the administration of the questionnaires. The sample comprised 583 employees and represented various demographic subgroups, viz. race, gender, age, job levels and language groups, in the organisations. A $58 \%$ response rate was achieved after the distribution of 1000 booklets $(N=583)$. The characteristics of the participants are reported in Table 1.

The gender distribution was $66,04 \%$ males and $29,67 \%$ females. A total of $4,29 \%$ of the participants did not indicate their gender. This compares well with the demographics of the general workforce in the chemical industry, the industry being predominantly male. The language representation of the sample also corresponds with the demographics of the area, with $72,51 \%$ of the respondents reporting either Afrikaans or English (a European language) as their home language, irrespective of racial category, and $25,10 \%$ of the respondents reporting Sesotho, isiZulu or isiXhosa (an African language) as their first language. The participants also represented the different job levels well, with $39,08 \%$ from the below-supervisory level, $30,72 \%$ from the first-line supervisory level, $6,83 \%$ from middlemanagement level and 2,22\% from senior management.

\section{Measuring instrument}

The 29-item Orientation to Life Questionnaire (OLQ-29) (Antonovsky, 1987) measures the three dimensions of the senseof-coherence construct, viz. comprehensibility, manageability

TABLE 1

Participant characteristics $(n=583)$

\begin{tabular}{|c|c|c|c|}
\hline & CATEGORY & FREQUENCY & PERCENTAGE \\
\hline \multirow[t]{5}{*}{ Age } & $<30$ & 99 & $16,98 \%$ \\
\hline & $30-39$ & 198 & $33,96 \%$ \\
\hline & $40-49$ & 182 & $31,22 \%$ \\
\hline & $50+$ & 91 & $15,61 \%$ \\
\hline & Missing & 13 & $2,23 \%$ \\
\hline \multirow[t]{3}{*}{ Gender } & Male & 385 & $66,04 \%$ \\
\hline & Female & 173 & $29,67 \%$ \\
\hline & Missing & 25 & $4,29 \%$ \\
\hline \multirow[t]{3}{*}{ Language } & European & 431 & $73,93 \%$ \\
\hline & African & 144 & $24,70 \%$ \\
\hline & Missing & 8 & $1,37 \%$ \\
\hline \multirow[t]{5}{*}{ Qualification } & School & 321 & $55,06 \%$ \\
\hline & Diploma & 143 & $24,53 \%$ \\
\hline & Degree & 70 & $12,01 \%$ \\
\hline & Postgraduate & 23 & $3,95 \%$ \\
\hline & Missing & 26 & $4,46 \%$ \\
\hline \multirow[t]{5}{*}{ Tenure } & $<5$ years & 134 & $22,98 \%$ \\
\hline & $5-14$ years & 166 & $28,47 \%$ \\
\hline & $15-24$ years & 205 & $35,16 \%$ \\
\hline & $25+$ years & 59 & $10,12 \%$ \\
\hline & Missing & 19 & $3,26 \%$ \\
\hline \multirow[t]{5}{*}{ Job Level } & Employees & 229 & $39,08 \%$ \\
\hline & Supervisors & 180 & $30,72 \%$ \\
\hline & Middle Managers & 40 & $6,83 \%$ \\
\hline & Senior Managers & 13 & $2,22 \%$ \\
\hline & Missing & 124 & $21,16 \%$ \\
\hline
\end{tabular}


TABLE 2

Orientation to Life Questionnaire (OLQ) construct validity

\begin{tabular}{|c|c|c|c|c|c|c|c|}
\hline MODEL & DESCRIPTION & $\operatorname{CMIN}\left(x^{2}\right)$ & $d f$ & CMIN/df & IFI & CFI & RMSEA \\
\hline Default & OLQ-29 1 factor (minus items 5 and 25) & 1452,94 & 324 & 4,48 & 0,67 & 0,67 & 0,08 \\
\hline I & OLQ-29 3 factors (minus items 5 and 25) & 1167,78 & 321 & 3,64 & 0,75 & 0,75 & $0,07^{*}$ \\
\hline II & OLQ-13 1 factor (all items) & 304,88 & 65 & 4,69 & 0,80 & 0,80 & 0,08 \\
\hline III & OLQ-13 1 factor (minus item 25) & 286,95 & 54 & 5,31 & 0,79 & 0,79 & 0,09 \\
\hline IV & OLQ-13 1 factor (minus items 5 and 25) & $145,98 \mathrm{a}$ & 44 & 3,32 & 0,89 & 0,89 & $0,06^{*}$ \\
\hline $\mathrm{v}$ & OLQ-13 1 factor (minus items 5 and 25; errors allowed to correlate between items 28 and 29) & $120,77 \mathrm{~b}$ & 43 & 2,81 & $0,92^{*}$ & $0,92^{*}$ & $0,06^{*}$ \\
\hline VI & OLQ-6 1 factor (items 8, 28 [mean], 12, 19 [comp.], 9, 29 [man.]) & $39,20 \mathrm{c}$ & 9 & 4,36 & $0,95^{*}$ & $0,95^{*}$ & 0,08 \\
\hline VII & $\begin{array}{l}\text { OLQ-6 } 3 \text { factors (items } 8,28 \text { [mean], 12, } 19 \text { [comp.], 9, } 29 \text { [man.]; errors allowed to correlate } \\
\text { between items } 9 \text { and 12) }\end{array}$ & $23,95 d$ & 8 & 3,00 & $0,97^{*}$ & $0,97^{*}$ & $0,06^{*}$ \\
\hline
\end{tabular}

* Significant fit; $\mathrm{IFI}>0,90 ; \mathrm{CFI}>0,90 ; \mathrm{RMSEA}<0,05$ [0,08] (AMOS, Arbuckle, 1997)

Note: $\mathrm{CFI}=$ Comparative Fit Index, IFI = Independent Fit Index, RMSEA = Root Mean Square Error of Approximation $\mathrm{a}, \mathrm{bc}, \mathrm{d}=$ significant change in $x^{2}(\mathrm{p}=0,000)$

and meaningfulness. Antonovsky (1993) suggests that the three theoretical components not be considered as sub-scales, since the items were first constructed with a facet-analysis design in order to vary the content systematically along a number of dimensions. Secondly, from his review of studies, Antonovsky concludes that the factor analysis of the scale is likely to produce a single-factor solution that would not reflect the three dimensions. Antonovsky (1993) reports the Cronbach alpha coefficients of the OLQ in 29 research studies varying between 0,85 and 0,91 . Test-retest reliability studies have found coefficients between 0,41 and 0,97 (Antonovsky, 1993). Responses are on seven-point semantic differentials, anchored by wordings related to the contents of each item.

\section{Statistical analysis}

Statistical analysis was carried out using the SPSS program (SPSS Inc., 2003). In the first step, means, standard deviations, skewness and kurtosis were determined to describe the data. To assess the fit of a proposed model, the structural equation model was performed using the maximum likelihood methods of AMOS (Arbuckle, 1997). A covariance matrix was used as the source for the input data. The data were tested for specific measurement and structural models and, according to recommended fit indices, a mixture of fit indices was used (Arbuckle, 1997; Hanse \& Engström, 1999). Among the fit indices produced by the AMOS program is the Chi-square statistic $\left(x^{2}\right)$, which is the test of absolute fit for the model. The $x^{2}$ value, however, is sensitive to sample size. Additional goodness-of-fit indices, such as the Goodness-of-Fit Index (GFI), the Adjusted Goodness-of-Fit Index (AGFI), the Normed Fit Index (NFI), the Comparative Fit Index (CFI), the Tucker-Lewis Index (TLI) and the Root Mean Square Error of Approximation (RMSEA), were therefore used in this study.

\section{Procedure}

The data were collected in a series of attempts applying various methods, such as group sessions, focused attempts in different areas and a mail questionnaire, to ensure a sufficient sample size.

\section{RESULTS}

SEM as implemented by the AMOS program was used to test the factorial models of the OLQ for the total group of respondents.

The default model comprised 27 of the original OLQ items, excluding items 5 and 25 identified for their low standard regression weights, and tested a one-factor model. Items 5 and 25 presented grammatical problems. Item 5: "Has it happened in the past that you were surprised by the behaviour of people whom you thought you know well?" is a grammatically poorly constructed question. Except for the tense error ("know" should be "knew"), the question could be simplified to: "Have you ever been surprised by the behaviour of people you thought you knew well?" Item 25 is equally problematic, as it is a double item with a statement followed by a question: "Many people - even those with a strong character-sometimes feel like losers in certain situations. How often have you felt this way in the past?" The statement part of the item is written in a parenthesis format and the question is complicated as a pleonasm - a word indicating the past tense ("felt") is followed by the extension "... in the past?"

Model I comprised the same 27 items as the default model, also excluding items 5 and 25, but was tested for a three-factor model. Taking cognisance of previously reported results on the OLQ-29, from Model II onwards the OLQ-13 (Antonovsky, 1987) was used, which initially included items 5 and 25 previously identified to have low standard regression weights. In Model III and Model IV, items 5 and 25 were excluded, resulting first in a 12-item version and later in an 11-item version of the OLQ. Model III and Model IV, with first item 25 (Model III) and then item 5 (Model IV) removed, were tested as single-factor models. In Model V, with items 5 and 25 removed, items 28 and 29 were also allowed to correlate $(r=0,263)$, since these items were identified with possible misspecifications and as loading on the same construct. A possible reason for the strong correlation between items 28 and 29 can be found in the first part of both items, which presents a strong emotional hint - "How often do you have the feeling..." - while the core of the question occurs only in the latter part of the item. With the position of the two items at the end of the questionnaire, the similarity between the items and their quick succession could result in a transfer from item 28 to item 29, evoking a similar response. Models VI and VII comprised six items, two items each selected for the three sense-of-coherence constructs, resulting in an OLQ-6 questionnaire. The six-item OLQ was tested as a one-factor model (Model VI) and a three-factor model (Model VII).

The construct equivalence for the successive models ranging from the OLQ-29, OLQ-13, OLQ-12, OLQ-11 to OLQ-6 was tested for fit, in which the $x^{2}$ values, degrees of freedom and probability value were considered. Additional goodness-offit statistics (Independent Fit Index [IFI], CFI and RMSEA) were considered further so that the global fit of each different model (Hanse \& Engström, 1999) could be assessed. The initial poor fit of the default model for the OLQ-29 resulted in further explorative analysis. The modification indices (MI) were considered as indicating possible misspecifications and item pairs with high MI values were allowed to correlate in subsequent models. Only items from the original OLQ-29 were used. Table 2 presents the sequential models as tested in an attempt to find the best possible fit.

The first step in the testing of construct equivalence was to use all the data in a one-factor model. The default model comprised the original OLQ-29 minus items 5 and 25, which showed low standard regression weights. The default model described rendered a $x^{2}$ value of $1452,94(d f=324 ; p=0,000)$. With the large 
TABLE 3

Descriptive statistics of the OLQ

\begin{tabular}{lrrrrr}
\hline ITEM & MEAN & SD & SKEWNESS & KURTOSIS & $\boldsymbol{\alpha}$ \\
\hline $\begin{array}{l}\text { Orientation to Life } \\
\text { Questionnaire (11 items) }\end{array}$ & 49,97 & 9,46 & $-0,27$ & 0,19 & $0,75^{*}$ \\
$\begin{array}{l}\text { Orientation to Life } \\
\text { Questionnaire (6 items) }\end{array}$ & 27,63 & 5,35 & $-0,54$ & 0,52 & $0,72^{*}$ \\
\hline
\end{tabular}

*Significant internal consistency > 0,70 (Nunnally \& Bernstein, 1994)

$x^{2}$ value relative to the degrees of freedom, together with an IFI value and CFI value lower than 0,90 , the model showed a poor fit. The same data were also tested in a three-factor model for the OLQ (Model I). The fit statistics remained unsatisfactory $\left(x^{2}=1167,78, d f=321 ; \mathrm{IFI}=0,67 ; \mathrm{CFI}=0,67\right.$; RMSEA $\left.=0,07\right)$, again showing a poor fit for the model.

Following the poor fits achieved and the negative variance reported, it was decided that the tested 13-item model would be applied using items $4,5,6,8,9,12,16,19,21,25,26,28$ and 29 . Model II, with all 13 items included, still yielded unsatisfactory fit parameters $\left(x^{2}=304,88, d f=65\right.$; IFI $=0,80$; CFI $=0,80$; RMSEA $=0,08$ ). Analysis of standard regression weights in Model II pointed towards item 25 with a negative coefficient, indicating a negative covariance that could affect the fit statistics. In the next model (Model III), item 25 was excluded based on the negative variance but the model still yielded unsatisfactory fit statistics $\left(x^{2}=286,95, d f=54 ; \quad\right.$ IFI $=0,79 ; \quad$ CFI $=0,79$; RMSEA $=0,09$ ). In consideration of the standardised regression weights achieved in Model III, item 5 was also identified with a low coefficient and item 5 was therefore also excluded in the next model. In Model IV, the fit statistics with items 5 and 25 removed resulted in an 11-item OLQ, which, in turn, resulted in near-sufficient fit parameters $\left(x^{2}=145,98, d f=44\right.$; IFI $=0,89$; $\mathrm{CFI}=0,89$; RMSEA $=0,06$ ). In the analysis of the correlation coefficients, items 28 and $29(r=0,263)$ were identified as possible misspecifications that correlated moderately, loading on the same construct that could influence the fit statistics negatively. In Model V, which had the same items as Model IV, the errors between items 28 and 29 were allowed to correlate in Model V, yielding acceptable fit statistics $\left(x^{2}=120,77, d f=43\right.$; IFI $=0,92$; CFI $=0,92$; RMSEA $=0,06$ ). Model V resulted in an 11-item questionnaire, with four items representing the comprehension factor, four items representing the manageability factor and three items representing the meaningfulness factor. The change in $x^{2}\left(\Delta x^{2}\right)$ against the change in degrees of freedom $(\Delta d f)$ from Model III to Model VII was significant.

In line with other research (Lundberg \& Nyström Peck, 1994; Schumann et al., 2003), further models were explored with the aim of reducing the number of items while maintaining Antonovsky's three-factor structure of sense of coherence and complying with basic psychometric principles, such as testing each factor with more than one question. In the analysis of the standard regression coefficients obtained with the previous model (Model VI), the items with the highest regression weights per factor were identified to be included in a six-item OLQ model. Model VI was then designed with items 8 and 28 (comprehension), items 12 and 19 (manageability) and items 9 and 29 (meaningfulness). Model VI yielded acceptable fit statistics $\left(x^{2}=32,20, d f=9\right.$; IFI $=0,95 ;$ CFI $=0,95$; RMSEA $\left.=0,08\right)$. The MI were considered as indicating possible misspecifications and items 9 and 12 were allowed to correlate in the next model, yielding better fit statistics in Model VII $\left(x^{2}=3,95, d f=8\right.$; IFI $=0,97$; CFI $=0,97$; RMSEA $=0,06$ ), confirming the fit of a six-item OLQ with two questions per factor (Antonovsky, 1987, 1993).

The descriptive statistics, alpha coefficients, skewness and kurtosis of the different factors and different OLQ models are presented in Table 3.

Both OLQ models presented satisfactory levels of internal consistency (OLQ-11 items $\alpha=0,75$; OLQ-6 items $\alpha=0,72$ ), with the Cronbach alpha coefficient being greater than 0,70 (Nunnally \& Bernstein, 1994). The Cronbach alpha coefficient is influenced by the number of items, partially explaining the reduction of the alpha coefficient value. Maintaining an alpha coefficient above 0,70 with less than half the original items indicates the model's consistency. The correlation between the two OLQ models is $0,93(p<0,01)$, confirming the similarity between the six-item and eleven-item OLQ versions.

In Table 4, the five demographic variables considered in terms of the variance in sense-of-coherence means are presented.

Table 4 shows that the variance reported for qualification and job level was on a statistically significant level $(p<0,01)$. The variance of the other demographic variables was not statistically significant (language, $p=0,03$; age, $p=0,02$; gender, $p=0,63$; tenure [years of service] $p=0,53$ ). The sense-of-coherence means increased with qualification level and significant differences were reported between the sense-of-coherence for the secondary-school group versus the sense-of-coherence for both the graduate and the postgraduate groups. The most significant differences among the sense-of-coherence means for sub-groups were reported for different job levels, where the sense-of-coherence means was found to increase with job level, with significant differences among all the job-level sub-groups.

TABLE 4

Analysis of variance - Orientation to Life Questionnaire (OLQ) (11 items)

\begin{tabular}{|c|c|c|c|c|c|c|c|c|c|}
\hline SOURCE & $\begin{array}{l}\text { SUM OF } \\
\text { SQUARES } \\
\text { (TYPE III) }\end{array}$ & $d f$ & $F$ & $p$ & MEAN & & & & \\
\hline & & & & & $<30$ years & $30-39$ years & $40-49$ years & $50+$ years & $p$ \\
\hline \multirow[t]{2}{*}{ Age } & 932,48 & 3 & 3,52 & 0,02 & $48,08 \mathrm{a}$ & 49,23 & $51,55 \mathrm{a}$ & 50,56 & 0,02 \\
\hline & & & & & Afrikaans/English & Africa & & & $p$ \\
\hline \multirow[t]{2}{*}{ Language } & 432,54 & 1 & 4,86 & 0,03 & 50,44 & 48,33 & & & 0,03 \\
\hline & & & & & Male & Female & & & $p$ \\
\hline \multirow[t]{2}{*}{ Gender } & 21,02 & 1 & 0,232 & 0,63 & 50,13 & 49,71 & & & 0,63 \\
\hline & & & & & Secondary & Diploma & Degree & Postgraduate & $p$ \\
\hline \multirow[t]{2}{*}{ Qualification } & 2055,53 & 3 & 7,89 & $0,00^{*}$ & $48,54 \mathrm{a}$ & 51,00 & $53,28 a$ & 54,65 & $0,00^{*}$ \\
\hline & & & & & $<5$ years & 5-14 years & $15-24$ years & $25+$ years & $p$ \\
\hline \multirow[t]{2}{*}{ Tenure } & 195,06 & 3 & 0,73 & 0,53 & 49,15 & 50,05 & 50,70 & 50,23 & 0,53 \\
\hline & & & & & Employee & Supervisor & Manager & Senior manager & $p$ \\
\hline Job Level & 2829,03 & 3 & 11,34 & $0,00^{*}$ & $47,78 \mathrm{ab}$ & $51,92 a$ & $55,10 \mathrm{bc}$ & $48,23 \mathrm{c}$ & $0,00^{*}$ \\
\hline
\end{tabular}

${ }^{*} p<0,00$ statistically significan

$\mathrm{a} b \mathrm{c}=$ significant comparison 
The mean sense of coherence for the senior-management group was, however, lower and in the order between the sense-ofcoherence mean of the employee group and the supervisor group. The differences between the sense-of-coherence means for the employees and their seniors (both supervisors and managers) were significant $(p<0,01)$, with the latter reporting successively stronger levels of sense of coherence. The difference between the means for the managers and the senior managers was also statistically significant, although the latter scored a lower mean sense of coherence than the managers.

\section{DISCUSSION}

The objectives of this study were to evaluate the construct equivalence of the OLQ in the chemical factory environment, to test the construct validity of a shortened version of the OLQ and to investigate differences in sense of coherence among demographic groups in a chemical factory environment.

The construct equivalence of the OLQ as applied to a sample of employees working in a chemical factory was confirmed by using AMOS (Arbuckle, 1997). The original OLQ-29 model showed poor fit statistics. Analysis of the data pointed to two problematic items, viz. items 5 and 25 . After removing items 5 and 25 , the fit statistics improved slightly but remained outside the recommended fit parameters. Based on previous research findings (Rothmann, 2000), the OLQ-13 was pursued for further model analysis. The OLQ-13 with items 6, 9, 25 and 29 (manageability), 4, 8, 16 and 28 (meaning) and 5, 12, 19, 21 and 26 (comprehension) subsequently produced better fit statistics than the original OLQ-29. Excluding the identified problematic items, items 5 and 25 rendered an 11-item OLQ, which resulted in satisfactory fit statistics and sufficient internal consistency $(\alpha=0,75)$ for sense of coherence as a one-factor construct.

To design an even shorter OLQ version than the OLQ-13 for possible inclusion in corporate-culture surveys, for instance, six items from the original OLQ-29 with the highest regression weights per factor were selected, with two items each per factor (OLQ-6): items 8 and 28 (meaning), 12 and 19 (comprehension) and 9 and 29 (manageability). Testing the construct validity of the OLQ-6 as a single-factor model, still with two questions per theoretical factor according to the theory by Antonovsky (1987), resulted in satisfactory fit statistics and a satisfactory level of internal consistency $(\alpha=0,72)$. The OLQ-6 also correlated highly with the OLQ-11 $(r=0,93)$, confirming that these two abbreviated OLQ versions could be used alternatively and would render statistically comparable results. The construct validity for the OLQ and specifically the shortened versions (OLQ-6 and OLQ-13) were confirmed, with sufficient levels of internal consistency for both the OLQ-6 and OLQ-11. The OLQ in the shorted formats is therefore confirmed to be statistically valid and reliable for use for different demographic groups in a chemical factory environment. Both the shortened OLQ versions can also be used within a theoretical three-factor model, supporting the theoretical conceptualisation of sense of coherence by Antonovsky (1987)

Another problem reported in literature pertains to the relationship between sense of coherence and demographic variables. Results reported either confirmed the relationship (Antonovsky \& Sagy, 2001; Hanse \& Engström, 1999; Larsson \& Kallenberg, 1996; Tuomi, Seitsamo, \& Huuhtanen, 1999) or refuted the relationship, with Holmberg et al. (2004) pointing to the low correlations between sense of coherence and sociodemographic variables, indicating the independence of sense of coherence from socio-demographic factors.

An analysis of OLQ data in this study did point out statistically significant differences among some demographic groups.
The mean sense of coherence for qualification level and job level was found to increase progressively and on statistically significant levels. The reported stability of sense of coherence from the age of 30 and beyond could not be confirmed. In line with sense of coherence as associated with the progressive and time-related mastering of work and life complexities, the mean sense of coherence was found to increase steadily with 10 -year intervals from the younger-than-30-year-old group to the 40-49-year-old group. The mean sense of coherence, however, levelled off beyond 50 years of age. The 30-year-old group from two decades ago is now the 50-year-old group. This points to sense of coherence as a result of the mastering of work and life complexities as a generation phenomenon rather than a chronological-age phenomenon, which concurs with the theory by Antonovsky (1987) about young adults' learning early in life.

The sense-of-coherence means for demographic variables where equity is expected, viz. language, gender and tenure, did not differ statistically on a significant level. The difference in the mean sense of coherence for the African-speaking groups was lower than for the Afrikaans/English-speaking group but not statistically significant. This can be attributed to historical reasons where, despite language differences - the predominant languages spoken in the area being either Sesotho (an African language) or Afrikaans - all language groups in the same area experienced similar learning experiences over time, thus contributing to their sense of coherence. The sense-of-coherence means among sub-groups with different years of service did not differ significantly.

This study contributes in presenting a version of the OLQ suitable for multilingual groups in the chemical factory environment. It does, however, have some limitations. The first limitation was that the scope of the measurements was restricted. The scope did not include other versions of the OLQ and comparisons were done based on other research published. The items of the OLQ were also used in the format available and as used in previous research. In hindsight and in view of the poor quality of the language found in some of the items, it is necessary to reconsider the wording of some of the items. The second limitation was that the sample size was limited, especially in the different language groups. The third limitation was that participants were sampled from the chemical industry and that the results could therefore not be generalised.

\section{RECOMMENDATIONS}

This study tested the construct validity of the original OLQ-29 as well as two short OLQ versions and explored the sense-ofcoherence differences for different demographic groups in a chemical factory environment. Although both the construct validity and the internal consistency of the shortened versions of the OLQ-11 and OLQ-6 were confirmed, a longitudinal study is recommended to assess the reliability and validity of the abbreviated OLQ formats. The psychometric results were satisfactory, confirming that, even if just on a theoretical level, it is possible to maintain the original three-factor structure of Antonovsky (1987) conceptualised for sense of coherence with items representing the three factors. From a psychometric point, the OLQ items must be revisited in terms of the quality of language used. At least on face value, a number of the items are plagued by language problems, viz. semantics, syntax and grammar, making it difficult for an English second-language speaker to comprehend.

The importance of positive life experiences, with all three components conceptualised by Antonovsky (1987) contributing to building a strong sense of coherence, is fundamental to the conceptualisation of sense of coherence and is well reported in 
literature. The inconclusive results pertaining to gender and years of service and statistically significant differences in the mean sense of coherence for age, qualification and job-level groups indicate the effect of positive life experiences on sense of coherence, regardless of gender or tenure. The implication for the chemical factory environment is that the development programmes for personnel can be tailor-made to facilitate and enhance the development of sense of coherence.

A cross-validation study should be considered in future studies of the construct validity of the OLQ.

\section{REFERENCES}

Agardh, E.E., Ahlbom, A., Andersson, T., Efendic, S., Grill, V., Hallqvist, J., Norman, A. \& Ostenson, C. (2003). Work stress and low sense of coherence are associated with type 2 diabetes in middle-aged Swedish women. Diabetes Care, 26, 719-724

Anderson, C.A. \& Riger, A.L. (1991). A controllability attribution model of problems in living: Dimensional and situational interactions in the prediction of depression and loneliness. Social Cognition 9(2), 149-181.

Antonovsky, A. (1979). Health, stress and coping: New perspective on mental and physical well-being. San Francisco, CA: JosseyBass.

Antonovsky, A. (1987). Antonovsky's sense of coherence scale and job attitudes: Three studies. South African Journal of Psychology, 31, 30-37.

Antonovsky, A. (1993). The structure and properties of the sense of coherence scale. Social Science E Medicine, 36, 725-733.

Antonovsky, H. \& Sagy, S. (2001). The development of a sense of coherence and its impact on responses to stress situations. The Journal of Social Psychology, 126(2), 213-225.

Arbuckle, J.L. (1997). Amos users' guide version 4.0. Chicago, IL: Smallwaters Corporation.

Bandura, A. (1982). Self-efficacy mechanism in human agency. American Psychologist, 37(2), 122-147.

Cilliers, F. (2001). The role of sense of coherence in group relations training. South African Journal of Industrial Psychology, 27(3), 13-18.

Colteryahn, K. \& Davis, P. (2004). Trends you need to know. Training and Development, 58(1), 28-36.

Eriksson, M. \& Lindström, B. (2005). Validity of Antonovsky's sense of coherence scale: A systematic review. Journal of Epidemiology and Community Health, 59, 460-466.

Feldt, T., Kivimäki, M., Rantala, A. \& Tolvanen, A. (2004). Sense of coherence and work characteristics: A cross-lagged structural equation model among managers. Journal of Occupational and Organizational Psychology, 77(3), 323-342.

Feldt, T., Leskinen, E., Kinnunen, U. \& Ruoppila, I. (2003). The stability of sense of coherence: Comparing two age groups in a 5-year follow-up study. Personality and Individual Differences, 35(5), 1151-1165.

Flannery, R.B., Perry, J.C., Penk, W.E. \& Flannery, G.J. (1994). Validating Antonovsky's sense of coherence scale. Journal of Clinical Psychology, 50(4), 575-577.

Geyer, S. (1997). Some conceptual considerations on the sense of coherence. Soc Sci Med, 44(12), 1771-1779.

Hanse, J.J. \& Engström, T. (1999). Sense of coherence and ill health among the unemployed and re-employed after closure of an assembly plant. Work E Stress, 13(3), 204-222.

Hittner, J.B. (2000). Novel methods for analyzing multifaceted personality scales: Sense of coherence and depression as an example. The Journal of Psychology, 134(2), 199-209.

Holmberg, S., Thelin, A. \& Stiernström, E. (2004). Relationship of sense of coherence to other psychosocial indices. European Journal of Psychological Assessment, 20, 227-236.

Javtokas, Z., Lindström, B. \& Zagminas, K. (2004). Sense of coherence and awakening pattern: Evidence from the population survey in Lithuania. Public Health, 3(26), 46-50.
Kobasa, S.C. (1979). Stressful life events, personality and health: An inquiry into hardiness. Journal of Personality and Social Psychology, 37(1), 1-11.

Larsson, G. \& Kallenberg, K.O. (1996). Sense of coherence, socio-economic conditions and health. Interrelationship in a nation-wide Swedish sample. European Journal of Public Health, 6(3), 175-180.

Larsson, G. \& Kallenberg, K.O. (1999). Dimensional analysis of sense of coherence using structural equation modelling. European Journal of Personality, 13(1), 51-61.

Lindfors, P., Lundberg, O. \& Lundberg, U. (2005). Sense of coherence and biomakers of health in 43-year-old women. International Journal of Behavioural Medicine, 12(2), 98-102.

Lundberg, O. \& Nyström Peck, M. (1994). Sense of coherence, social structure and health. European Journal of Public Health, 4(4), 252-257.

Nilsson, B., Holmgren, L., Stegmayer, B. \& Westman, G. (2003). Sense of coherence - stability over time and relation to health, disease, and psychosocial changes in a general population: A longitudinal study. Scandinavian Journal of Public Health, 31(4), 297-304.

Nunnally, J.C. \& Bernstein, I.H. (1994). Psychometric theory. 3rd edition. New York: McGraw-Hill.

Nygren, B., Aléx, L., Jonsén, E., Gustafson, Y., Norberg, A. \& Lundman, B. (2005). Resilience, sense of coherence, purpose in life and self-transcendence in relation to perceived physical and mental health among the oldest old. Aging and Mental Health, 9(4), 354-362.

Olsson, M.B. \& Hwang, C.P. (2002). Sense of coherence in parents of children with different developmental disabilities. Journal of Intellectual Disability Research, 46(7), 548-559.

Ortlepp, K. \& Friedman, M. (2001). The relationship between sense of coherence and indicators of secondary traumatic stress in non-professional trauma counsellors. South African Journal of Psychology, 31(2), 38-44.

Petrie, K. \& Brook, R. (1992). Sense of coherence, self-esteem, depression and hopelessness as correlates of reattempting suicide. British Journal of Clinical Psychology, 31(3), 293-300.

Randall, S.J. (2006). A validation study of the sense of coherence scale using two South African samples. Unpublished dissertation. Faculty of Humanities, University of the Witwatersrand, Johannesburg.

Rothmann, S., Jackson, L.T.B. \& Kruger, M.M. (2003). Burnout and job stress in a local government: The moderating effect of sense of coherence. South African Journal of Industrial Psychology, 29(4), 52-60.

Rothmann, S. (2000). Sense of coherence, locus of control and job satisfaction. Paper presented at the 27th International Congress of Psychology, Stockholm.

Rothmann, S., Steyn, L.J. \& Mostert, K. (2005). Job stress, sense of coherence and work wellness in an electricity supply organization. South African Journal of Business Management, 36(1), 55-63.

Schumann, A., Hapke, U., Meyer, C., Rumpf, H.J. \& John, U. (2003). Measuring sense of coherence with only three items: A useful tool for population surveys. British Journal of Health Psychology, 8(4), 409-421.

Shaughnessy, J.J. \& Zechmeister, E.B. (1997). Research methods in psychology. (4th Ed.). New York: McGraw-Hill.

Sjöström, H., Langius-Eklof, A. \& Hjertberg, R. (2004). Wellbeing and sense of coherence during pregnancy. Acta Obstetricia Gynecologica Scandinavica, 83(12), 1112-1118.

Smith, P.M., Breslin, F.C. \& Beaton, D.E. (2003). Questioning the stability of sense of coherence: The impact of socio-economic status and working conditions in the Canadian population. Journal of Managerial Psychology, 18, 557-572.

Snekkevik, H., Anke, A.G.W., Stanghelle, J.K. \& Fugl-Meyer, A.R. (2003). Is sense of coherence stable after multiple trauma? Clinical Rehabilitation, 17(4), 443-453.

Söderfeldt, M., Söderfeldt, B., Ohlson, C., Theorell, T. \& Jones, I. (2000). The impact of sense of coherence and high-demand/ low-control job environment on self-reported health, 
burnout and psycho-physiological stress indicators. Work $\mathcal{E}$ Stress, 14(1), 1-15.

SPSS (2003). SPSS 12.0 for Windows. Chicago, IL: SPSS Incorporated.

Strauser, D.R. \& Lustig, D.C. (2003). The moderating effect of sense of coherence on work adjustment. Journal of Employment Counselling, 40(3), 129-140.
Tuomi, K., Seitsamo, J. \& Huuhtanen, P. (1999). Stress management, aging, and disease. Experimental Aging Research, 25(4), 353-358.

Volanen, S.M., Lahelma, E., Silventoinen, K. \& Suominen, S. (2004). Factors contributing to sense of coherence among men and women. European Journal of Public Health, 14(3), 322-330. 